\title{
CLASSES OF MAXIMUM NUMBERS ASSOCIATED WITH CERTAIN SYMMETRIC EQUATIONS IN $n$ RECIPROCALS*
}

\author{
BY NORMA STELFORD AND H. A. SIMMONS
}

1. Introduction. In an article dealing with this subject Simmons $\dagger$ stated without proof two general theorems whose proofs are to be obtained by making certain modifications in the theory of his article, which will be referred to in the sequel as I (for paper I). These theorems will be stated after a few definitions from I are recalled.

Kellogg solution. If a solution $x \equiv\left(x_{1}, x_{2}, \cdots, x_{n}\right)$ of any given symmetric equation in $n$ reciprocals is obtained by minimizing the variables $x_{1}, x_{2}, \cdots, x_{n-1}$ (all positive integers) in this order, one at a time, we shall denote it by $w$ and call it the Kel$\operatorname{logg}$ solution of the given equation. Thus $x \equiv(2,3,6)$ is the Kellogg solution $w$ of the equation $x_{1}^{-1}+x_{2}^{-1}+x_{3}^{-1}=1$.

E-solution. A solution $x \equiv\left(x_{1}, x_{2}, \cdots, x_{n}\right)$ of a given symmetric equation in $n$ reciprocals is called an $E$-solution if $x_{1}$, $x_{2}, \cdots, x_{n-1}$ are positive integers and $x_{1} \leqq x_{2} \leqq \cdots \leqq x_{n}$.

Polynomial $P(x)$. Let $P\left(x_{1}, x_{2}, \cdots, x_{n}\right) \equiv P(x)$ be any polynomial which is symmetric in the $n$ variables $x_{i}$, contains one or more positive coefficients and no negative coefficient, and is not identically a constant.

$\Sigma_{i, j}(x)$. With $i \geqq 0$ and $j$ equal to integers, we let $\Sigma_{i, j}(x)$ stand for the $j$ th elementary symmetric function of the $i$ variables $x_{1}, x_{2}, \cdots, x_{i}$; with the customary understanding that

$$
\Sigma_{i, j}(x)\left\{\begin{array}{l}
\equiv 0 \text { when } i<j \text { and also when } j<0, \\
\equiv 1 \text { when } j=0 .
\end{array}\right.
$$

We now state the two theorems referred to above with the numbering of $I$.

TheOREM 4. If in the equation

* Presented to the Society, April 6, 1934. 907.

$\dagger$ See H. A. Simmons, Transactions of this Society, vol. 34 (1932), pp. 876- 


$$
\begin{aligned}
\Sigma_{n, r}(1 / x) & +\lambda_{r+1} \Sigma_{n, r+1}(1 / x)+\lambda_{r+2} \Sigma_{n, r+2}(1 / x)+\cdots \\
& +\lambda_{s} \Sigma_{n, 8}(1 / x)=b / a, \quad a \equiv[(c+1) b-1],
\end{aligned}
$$

where $b$ and $c$ are positive integers, either $\lambda_{p}=1,(p=r+1, \cdots, s)$, or $\lambda_{r+1}$ is an integer $\geqq 0$ and $\lambda_{p}=0,(p=r+2, \cdots, s)$, the largest number that exists in any $E$-solution of the resulting equation (21) is the $w_{n}$ of the corresponding solution $w$ defined by the recurrence relations

$$
\begin{aligned}
w_{p}= & 1, \quad(p=1, \cdots, r-1), \quad w_{r}=c+1, \\
w_{p+1}= & a\left[\Sigma_{p, p-r+1}(w)+\lambda_{r+1} \Sigma_{p, p-r}(w)+\lambda_{r+2} \Sigma_{p, p-r-1}(w)\right. \\
& \left.+\cdots+\lambda_{s} \Sigma_{p, p-s+1}(w)\right]+1, \quad(p=r, \cdots, n-2), \\
w_{n}= & a\left[\Sigma_{n-1, n-r}(w)+\lambda_{r+1} \Sigma_{n-1, n-r-1}(w)+\lambda_{r+2} \Sigma_{n-1, n-r-2}(w)\right. \\
& \left.+\cdots+\lambda_{s} \Sigma_{n-1, n-s}(w)\right] .
\end{aligned}
$$

Furthermore, in each of these cases, $w_{n}$ appears in but one E-solution of the equation (21) in question.

THEOREM 5. In each of the two cases of Theorem 4 , if $X$ is an E-solution of equation (21) and is different from the w of that equation, then $P(X)<P(w)$.

Here we shall extend all theorems of I that relate to maximum numbers by considering cases in which the right members, $b / a$, of the equations in question are much more general than they were in I. For (a very simple) example, consider

$$
\frac{1}{x_{1}}+\frac{1}{x_{2}}+\frac{1}{x_{3}}=\frac{3}{3 c-2},
$$

where $c \geqq 3$ is odd. The Kellogg solution of this equation is readily found to be $x=w$, where $w_{1}=c, w_{2}=2^{-1}\left(3 c^{2}-2 c+1\right)$, $w_{3}=(3 c-2) w_{1} w_{2}$. We show later that the theorems above hold for this solution $w$. This conclusion does not follow from I because $(3 /(3 c-2))-\left(1 / w_{1}\right)$ is not a unit fraction, while in I, $(b / a)-\left(1 /\left(w_{1} \cdots w_{r}\right)\right),(r=1, \cdots, n-1)$, was required to be a unit fraction. Our new theorems are stated in $\$ \S 6,9,10$.

We divide the portion of this paper from $\$ 2$ to the end into two parts. In Part $1, \S \S 2$ to 5 , we prove a case of Theorem 4 ; in Part 2, we develop our extensions as far as seems desirable.

Except where the contrary is stated, we employ here the definitions and notation of I. The analogs of equation ( $i$ ) and 
Theorem $i$ of $\mathrm{I}$, if written here, are denoted by ( $i \mathrm{a})$ and Theorem $i$ a, respectively, and proofs that are to be obtained as were analogous proofs of $\mathrm{I}$ are omitted. Consequently, in Part 1 we only state our modifications of certain definitions and lemmas and prove inequality (46a).

\section{Part 1. Proof of one Case of Theorem 4}

2. New Relations. We shall prove the part of Theorem $4, \S 23$ of I, for which $\lambda_{r+1}=\lambda_{r+2}=\cdots=\lambda_{s}=1$, that is, the case where equation (21) is

$$
\phi_{n}(1 / x)=b / a,
$$

where $\phi_{n}(1 / x) \equiv \Sigma_{n, r}(1 / x)+\Sigma_{n, r+1}(1 / x)+\cdots+\Sigma_{n, s}(1 / x)$, and $r, s, n$ are positive integers such that $r<s \leqq n$.

If in the last displayed equation $n$ is replaced by $p, r \leqq p \leqq n$, our definition of $\phi_{p}(1 / x)$ is obtained.

The analogs here of (26), (28), (29), (30), (32) of I are as follows.

(26a)

$$
\begin{aligned}
w_{p}= & 1, \quad(p=1, \cdots, r-1), \quad w_{r}=c+1, \\
w_{p+1}= & a\left[\Sigma_{p, p-r+1}(w)+\Sigma_{p, p-r}(w)+\cdots\right. \\
& \left.+\Sigma_{p, p-s+1}(w)\right]+1, \quad(p=r, \cdots, n-2), \\
w_{n}= & a\left[\Sigma_{n-1, n-r}(w)+\Sigma_{n-1, n-r-1}(w)+\cdots\right. \\
& \left.+\Sigma_{n-1, n-s}(w)\right] . \\
\phi_{p}(1 / w)= & \left(b w_{1} \cdots w_{p}-1\right) /\left(a w_{1} \cdots w_{p}\right), \\
& \quad(p=r, \cdots, n-1 ; \operatorname{see}(27) \text { of I }) . \\
\phi_{p}(1 / X) \leqq & \left(b X_{1} \cdots X_{p}-1\right) /\left(a X_{1} \cdots X_{p}\right), \\
& (p=r, \cdots, n-1),
\end{aligned}
$$

which is to be proved as was (29) of I.

$$
\begin{array}{rrr}
\text { (30a) } & \phi_{n-1}(1 / X) \leqq \phi_{n-1}(1 / w), & (1 \leqq r<s \leqq n) . \\
& \Sigma_{n-1, r-1}(1 / X)+\Sigma_{n-1, r}(1 / X)+\cdots+\Sigma_{n-1,8-1}(1 / X) \\
\text { (32a) } \quad<\Sigma_{n-1, r-1}(1 / w)+\Sigma_{n-1, r}(1 / w)+\cdots+\Sigma_{n-1,8-1}(1 / w) \\
& \quad(1 \leqq r<s \leqq n) .
\end{array}
$$


3. New Definitions of Sets $\sigma, \tau$, and of the Transformation. $\operatorname{Sets}^{*} \sigma, \tau$. Let $\lambda$ be a fixed positive integer such that $r \leqq \lambda \leqq n$, where $r$ and $n$ are as they are defined for (21a). We shall call $x_{1} \ldots \lambda$ a set $\sigma$ (relative to the $w$ of (26a)) if, and only if, $\phi_{p}(1 / x) \leqq \phi_{p}(1 / w)$ for every positive integer $p$ such that $r \leqq p \leqq \lambda$. We shall call $x_{1} \ldots(\lambda+1)$ a set $\tau$ if, and only if, with $\lambda$ a positive integer such that $r \leqq \lambda \leqq n-2, x_{1} \ldots(\lambda+1)$ is not, and $x_{1} \ldots \lambda$ is, a set $\sigma$.

The new transformation. + Suppose $X_{1}^{\prime} \ldots$ contains at least one element of each of the classes $A$ and $B$ defined in I. Then we define our transformation of $X_{1} \ldots \nu$ into a new set $X_{1}{ }^{\prime} \ldots$, by $\left(t_{3 \mathrm{a}}\right)$ or $\left(t_{4 \mathrm{a}}\right)$ :

$$
\begin{gathered}
\left(t_{3 \mathrm{a}}\right) \quad X_{p}^{\prime}=X_{p}\left(p \neq q_{1}, q, p \leqq \nu\right), X_{q_{1}}^{\prime}=w_{q_{1}}, \\
\phi_{\nu}\left(1 / X^{\prime}\right)=\phi_{\nu}(1 / X) ; \\
\left(t_{4 \mathrm{a}}\right) \quad X_{p}^{\prime}=X_{p}\left(p \neq q_{1}, q, p \leqq \nu\right), X_{1 q}^{\prime}=w_{1 q}, \\
\phi_{\nu}\left(1 / X^{\prime}\right)=\phi_{\nu}(1 / X),
\end{gathered}
$$

according as $\left(t_{3 \mathrm{a}}\right)$ requires $X_{i q}^{\prime}$ to be not greater than $w_{i q}$ or greater than $w_{1}$, respectively.

4. Analogs of Lemmas 6, 7, 8 .

LEMma 6a. (i) If $X_{1} \ldots k_{k}$ is a set $\tau, X_{1} \ldots{ }_{k}$ is transformable. $\neq$ (ii) If $X_{1} \ldots{ }_{k}$ is a set $\tau$ or a transformable set $\sigma$ for which $r<k \leqq n$, and if $t$ is a positive integer, application of (33a) with $\nu=k$ to $X_{1} \ldots{ }_{k}$ yields $a$ set $X_{1}^{\prime} \ldots{ }_{k}$ such that $(34 \mathrm{a})$, identical with (34), is true, and

$$
\phi_{p}\left(1 / X^{\prime}\right) \leqq \phi_{p}(1 / w), \text { for } p=r, \cdots,{ }_{1} q-1 ;
$$

[(36a) is not needed];

$$
\begin{aligned}
& \phi_{p}\left(1 / X^{\prime}\right)<\phi_{p}(1 / X), \text { for } p={ }_{1} q, \cdots, k-1 ; \\
& \phi_{p}\left(1 / X^{\prime}\right)=\phi_{p}(1 / X), \text { for } p=k .
\end{aligned}
$$

LEMmA 7a. If $s_{1}, s_{2}, \lambda$ are integers $\geqq 0$ and $s_{1} \geqq s_{2}, \lambda>0$, then

$$
\left(1^{s_{1}}\right)\left(1^{s_{2}}\right)>\left(1^{s_{1}+\lambda}\right)\left(1^{s_{2}-\lambda}\right) \text {. }
$$

\footnotetext{
* See pp. 889-890 of I.

$\dagger$ See p. 891 of $I$.

$\ddagger$ See p. 891 of I.
} 
In proving the next lemma by our method, Lemma 7 a would be used.

Lemma 8a. If $u, v, \gamma, t$ are integers with $u>v \geqq \gamma \geqq 1,0 \leqq t$ $\leqq(\gamma-1)$, and $x_{i}>0$ for $i=1, \cdots, u$, then

$$
\Sigma_{u, \gamma}(1 / x) \Sigma_{v, t}(1 / x)>\Sigma_{u, t}(1 / x) \Sigma_{v, \gamma}(1 / x) .
$$

5. Analog of Relation (46) of I. From (37a) and (38a) one may obtain (46a) below as (46) was obtained from (37) and (38) of I:

$$
\begin{aligned}
& {\left[\Sigma_{k-2, r-1}^{\prime}+\Sigma_{k-2, r}^{\prime}+\cdots+\Sigma_{k-2, s-1}^{\prime}\right]\left[\Sigma_{p-2, r-2}^{\prime}\right.} \\
& \left.\quad+\Sigma_{p-2, r-1}^{\prime}+\cdots+\Sigma_{p-2, s-2}^{\prime}\right] \\
& \quad>\left[\Sigma_{k-2, r-2}^{\prime}+\Sigma_{k-2, r-1}^{\prime}+\cdots+\Sigma_{k-2, s-2}^{\prime}\right]\left[\Sigma_{p-2, r-1}^{\prime}\right. \\
& \left.\quad+\Sigma_{p-2, r}^{\prime}+\cdots+\Sigma_{p-2, s-1}^{\prime}\right] .
\end{aligned}
$$

With

$$
\begin{aligned}
& c_{1} \equiv \Sigma_{k-2, r-1}^{\prime}+\Sigma_{k-2, r}^{\prime}+\cdots+\Sigma_{k-2, s-2}^{\prime}, \\
& c_{2} \equiv \Sigma_{p-2, r-1}^{\prime}+\Sigma_{p-2, r}^{\prime}+\cdots+\Sigma_{p-2,8-2}^{\prime},
\end{aligned}
$$

(46a) may be written

$$
\left(\Sigma_{k-2,8-1}^{\prime}+c_{1}\right)\left(\Sigma_{p-2, r-2}^{\prime}+c_{2}\right)>\left(\Sigma_{k-2, r-2}^{\prime}+c_{1}\right)\left(\Sigma_{p-2,8-1}^{\prime}+c_{2}\right) .
$$

Proof of (46a). That this inequality is true follows from the inequalities $I_{1}, I_{2}, I_{3}$ below, which we presently prove. In arriving at (46a), one finds that it is to hold under the hypotheses $1 \leqq r \leqq 1 q \leqq p<k$, with ${ }_{1} q \geqq 2$. These relations are understood to hold in this proof.

$$
\begin{aligned}
c_{1} \Sigma_{p-2, r-2}^{\prime} & >c_{2} \Sigma_{k-2, r-2}^{\prime}, \\
c_{2} \Sigma_{k-2, s-1}^{\prime} & \geqq c_{1} \Sigma_{p-2,8-1}^{\prime}, \\
\Sigma_{k-2,8-1}^{\prime} \Sigma_{p-2, r-2}^{\prime} & \geqq \Sigma_{k-2, r-2}^{\prime} \Sigma_{p-2,8-1}^{\prime} .
\end{aligned}
$$

The terms in $c_{1}$ are $\Sigma_{k-2, \lambda}^{\prime},(\lambda=r-1, \cdots, s-2)$; those in $c_{2}, \Sigma_{p-2, \lambda}{ }^{\prime}$. Hence $\left(\mathrm{I}_{1}\right),\left(\mathrm{I}_{2}\right)$ may be written

$$
\begin{aligned}
& \Sigma_{k-2, \lambda}^{\prime} \Sigma_{p-2, r-2}^{\prime}>\Sigma_{k-2, r-2}^{\prime} \Sigma_{p-2, \lambda}^{\prime}, \\
& \Sigma_{k-2,8-1}^{\prime} \Sigma_{p-2, \lambda}^{\prime} \geqq \Sigma_{k-2, \lambda}^{\prime} \Sigma_{p-2,8-1}^{\prime},
\end{aligned}
$$

* $\Sigma_{i, j}^{\prime} \equiv \Sigma_{i, j}^{\prime}(1 / X)$. 
respectively. If $p-2 \geqq \lambda,\left(\mathrm{I}_{1}^{\prime}\right)$ holds by Lemma 8 a with $u=k-2$, $v=p-2, \gamma=\lambda$, and $t=r-2$. If $(p-2)<\lambda$, then since $p \geqq r$ the left side of $\left(I_{1}^{\prime}\right)$ is positive and the right side is zero, so that $\left(\mathrm{I}_{1}^{\prime}\right)$ holds. Consider $\left(\mathrm{I}_{2}^{\prime}\right)$. If $(p-2) \geqq(s-1),\left(\mathrm{I}_{2}^{\prime}\right)$ holds with $>$ by Lemma $8 \mathrm{a}$, with $u=k-2, v=p-2, \gamma=s-1, t=\lambda$. If $(p-2)$ $<(s-1)$, the right side of $\left(\mathrm{I}_{2}^{\prime}\right)$ is zero and the left side is surely not negative. Therefore $\left(\mathrm{I}_{2}^{\prime}\right)$ holds with $\geqq$. The proof for $\left(\mathrm{I}_{2}\right)$ is a special case of that for $\left(\mathrm{I}_{2}^{\prime}\right)$.

\section{Part 2. Extensions of Previous Theory}

6. A New Theorem on Equations of the Form $\Sigma_{n, 1}(1 / x)=b / a$. The article I contains results for equations of this type only when $a=[(c+1) b-1]$ and $b, c$ are positive integers. This case is covered by taking $\mu=1$ in the new Theorem A below.

Theorem A. Suppose $a, b$ and $\mu, \mu \leqq(n-1)$, are given positive integers, with $a$ and $b, b \leqq a$, relatively prime, and that there exists a set of $n$ numbers $w \equiv\left(w_{1}, w_{2}, \cdots, w_{n}\right)$ with the following properties:

$\left(1^{\circ}\right)$ it is an E-solution of the equation $\Sigma_{n, 1}(1 / x)=b / a$;

$$
\frac{1}{x_{1}}+\frac{1}{x_{2}}+\cdots+\frac{1}{x_{n}}=\frac{b w_{1} \cdots w_{p}-1^{*}}{a w_{1} \cdots w_{p}}
$$

for every positive integral value of $p$ for which $\mu \leqq p \leqq(n-1)$ and for no smaller value of $p$;

$\left(3^{\circ}\right)$ if $x=X$, where $X \neq w$, is an E-solution of the equation in $\left(1^{\circ}\right)$, then for every positive integral value of $p \leqq \mu$ for which $X_{1} \cdots p$ $\neq w_{1} \ldots p$, the inequality $\Sigma_{p, 1}(1 / X)<\Sigma_{p, 1}(1 / w)$ holds.

For such a set w the following conclusions hold:

(i) $w_{n}$ is the largest number that exists in any E-solution of the equation in $\left(1^{\circ}\right)$ and $w_{n}$ appears in no E-solution of this equation except w;

(ii) $P(x)<P(w) . \dagger$

Proof of (i). It follows from hypotheses $\left(j^{\circ}\right),(j=1,2,3)$, that the $w$ described in Theorem $\mathrm{A}$ is the Kellogg solution of the equation in $\left(1^{\circ}\right)$. This fact will be used in the rest of the proof of (i), which we presently make by considering separately the following cases: $(\alpha) \mu=n-1 ;(\beta) \mu=n-2 ;(\gamma) \mu \leqq(n-3)$.

* See equation (28) of I, with $r=1$.

† See p. 887 of I. 
$(\alpha)$ In this trivial case, $\left(3^{\circ}\right)$ requires that conclusion (i) be true.

( $\beta$ ) If $X_{1} \ldots \mu=w_{1} \ldots \mu$, since $X \not \neq w$ is an $E$-solution of the equation in $\left(1^{\circ}\right)$ while $w$ is its Kellogg solution, $X_{\mu+1} \equiv X_{n-1}>w_{n-1}$, and (i) is true. If $X_{1} \ldots{ }_{\mu} \neq w_{1} \ldots{ }_{\mu}$, we reach the desired conclusion as follows. From the hypotheses $\left(j^{\circ}\right),(j=1,2,3)$, and the assumption that $X_{1} \ldots{ }_{\mu} \neq w_{1} \ldots \mu$, with $\mu=n-2$, we have

$$
\Sigma_{p, 1}(1 / X) \leqq \Sigma_{p, 1}(1 / w), \quad(p=1, \cdots, \mu-1) ;
$$

$$
\begin{aligned}
& \Sigma_{n-2,1}(1 / X)<\Sigma_{n-2,1}(1 / w) \equiv \frac{b w_{1} \cdots w_{n-2}-1}{a w_{1} \cdots w_{n-2}} \\
& \Sigma_{n-1,1}(1 / X) \leqq \frac{b X_{1} \cdots X_{n-1}-1}{a X_{1} \cdots X_{n-1}}, \quad[\text { see (29) of } \mathrm{I}]
\end{aligned}
$$

and we wish to contradict the assumption which we now make, namely that

$$
\Sigma_{n-1,1}(1 / X) \geqq \Sigma_{n-1,1}(1 / w) \equiv \frac{b w_{1} \cdots w_{n-1}-1}{a w_{1} \cdots w_{n-1}} .
$$

Relations (2) and (3) imply that

$$
X_{1} \cdots X_{n-1} \geqq w_{1} \cdots w_{n-1}
$$

(1) and (3), that $X_{1} \cdots(n-1)$ is transformable. Since $X$ is an $E$ solution, it follows now that when exhaustive applications of (33a) for $X_{1} \ldots(n-1)$ are made, the following relations hold [see (53) of I]:

$$
X_{1} \cdots X_{n-1}<X_{1}^{\prime} \cdots X_{n-1}^{\prime} \leqq w_{1} \cdots w_{n-1} .
$$

Since (4) and (5) are contradictory, we conclude that (3) is false. Consequently conclusion (i) holds in case $(\beta)$.

$(\gamma)$ The demonstration that is required here is essentially the same as that given under $(\beta)$ for the case $X_{1} \ldots \neq \neq w_{1} \ldots \mu$; induction integers $k,(k+1)$, where $\mu \leqq k \leqq(n-2)$, here take the place of $(n-2),(n-1)$, respectively, there. Hence conclusion (i) holds in case $(\gamma)$.

Proof of (ii). This result can be reached by the method that was employed in proving Theorem 3 of I, since inequalities analogous to those in (53) of I hold here. Hence Theorem A is true. 
7. An Example of Takenouchi. The Takenouchi equation*

$$
\frac{1}{x_{1}}+\frac{1}{x_{2}}+\frac{1}{x_{3}}=\frac{5}{11}
$$

has, as we presently prove, no solution $w$ of the type hypothesized in Theorem A. For the Kellogg solution of (6) is $X=w$ $=(3,9,99)$, and it is easily seen that for this $X$ there does not exist an integer $\mu$ of the type specified in $\left(2^{\circ}\right)$. One may also negate the existence here of the $w$ of Theorem A by observing with Takenouchi that the set $(4,5,220)$ satisfies (6), while $\left(4^{-1}+5^{-1}\right)>\left(3^{-1}+9^{-1}\right)$, so that $(3,9)=\left(w_{1}, w_{2}\right)$ does not accord with hypothesis $\left(3^{\circ}\right)$.

8. Corollaries of Theorem A. We have seen in I that when $a=[(c+1) b-1]$, the $w$ of Theorem A always exists (case $\mu=1$ ). When $a \neq[(c+1) b-1]$ there are, as we point out in the corollaries below, numerous cases for which the $w$ of Theorem A exists with $\mu=2$. In cases where $\mu>2$, we have no better method than that of trial and error for testing a given $E$-solution $X$ to see whether it satisfies hypothesis $\left(3^{\circ}\right)$. However, when $\mu=2$ the following Auxiliary Theorem shows that hypothesis $\left(3^{\circ}\right)$ is always satisfied when $\left(1^{\circ}\right)$ and $\left(2^{\circ}\right)$ are. For Theorem A only the case $r=1, \lambda_{r+1}=\lambda_{2}=0$ of this Auxiliary Theorem is needed; other cases of it are similarly useful in connection with Theorem $i \mathrm{a},(i=2,3,4,5)$, of I.

Auxiliary Theorem. Let $X$ be an E-solution of the equation

$$
\Sigma_{n, r}(1 / x)+\lambda_{r+1} \Sigma_{n, r+1}(1 / x)+\cdots+\lambda_{s} \Sigma_{n, s}(1 / x)=b / a,
$$

where $X \neq w$, w being the Kellogg solution of this equation; the $\lambda_{i},(i=r+1, \cdots, s, s>r)$, are integers $\geqq 0 ; n \geqq r+2 ; \dagger$ and $a, b$ are as in Theorem A except that we now suppose that

and that

$$
\frac{1}{w_{1} \cdots w_{r}} \neq \frac{b w_{1} \cdots w_{r}-1}{a w_{1} \cdots w_{r}}
$$

* See p. 92 of loc. cit. in footnote 3, p. 876, of I.

$\dagger$ This restriction insures the presence of at least two terms free of $x_{n}$ in the left member of the equation just displayed. When $n=r+1$, Theorem A has no content beyond the case $\mu=1$, which was handled in I. 


$$
\begin{gathered}
\frac{1}{w_{1} \cdots w_{r}}+\frac{1}{w_{r+1}}\left[\Sigma_{r, r-1}(1 / w)+\frac{\lambda_{r+1}}{w_{1} \cdots w_{r}}\right] \\
=\frac{b w_{1} \cdots w_{r+1}-1}{a w_{1} \cdots w_{r+1}} .
\end{gathered}
$$

Then we conclude that $\left(X_{1} \ldots{ }_{(r+1)}\right.$ is a set $\sigma$ in the sense that $)$

$$
\begin{gathered}
\frac{1}{X_{1} \cdots X_{r}} \leqq \frac{1}{w_{1} \cdots w_{r}} ; \\
\frac{1}{X_{1} \cdots X_{r}}+\frac{1}{X_{r+1}}\left[\Sigma_{r, r-1} \frac{1}{X}+\frac{\lambda_{r+1}}{X_{1} \cdots X_{r}}\right]<\frac{b w_{1} \cdots w_{r+1}-1}{a w_{1} \cdots w_{r+1}} .
\end{gathered}
$$

The proof of this theorem can be made by reasoning of the type that was used on p. 897 of I. Each of the three corollaries below is a case $\mu=2$ of Theorem A.

COROllary 1. For the equation $\Sigma_{n, 1}(1 / x)=5 / 17$, with $n>2$, the $w$ of Theorem $\mathrm{A}$ is $w_{1}=4, w_{2}=23, w_{i+1}=17 w_{1} \cdots w_{i}+1$, $(i=2, \cdots, n-2), w_{n}=17 w_{1} \cdots w_{n-1}$.

Corollary 2. For the equation $\Sigma_{n, 1}(1 / x)=3 /(3 c-2)$, with $n>2$, if $c \geqq 3$ is odd, the w of Theorem $\mathrm{A}$ is defined by the equations $w_{1}=c, \quad w_{2}=2^{-1}\left(3 c^{2}-2 c+1\right), \quad w_{i+1}=(3 c-2) w_{1} \cdots w_{i}+1$, $(i=2, \cdots, n-2), w_{n}=(3 c-2) w_{1} \cdots w_{n-1}$.

CoRollary 3. For the equation $\Sigma_{n, 1}(1 / x)=b / a$, with $n>2$ and $a \equiv[(c+1) b-2]$, if $b>1$ is odd and $c$ is even, the set $w$ of Theorem A is defined by $w_{1}=c+1, w_{2}=2^{-1}[a(c+1)+1]$, $w_{i+1}=a w_{1} \cdots w_{i}+1,(i=2, \cdots, n-2), w_{n}=a w_{1} \cdots w_{n-1}$.

9. Generalizations of Theorem 2, 3 of $\mathrm{I}$.

TheOREm 2a. Suppose $a, b$ and $\mu$, where $r \leqq \mu \leqq(n-1)$, are given positive integers, with $a$ and $b, b \leqq a$, relatively prime, and that there exists a set of $n$ numbers $w \equiv\left(w_{1}, \cdots, w_{n}\right)$ with the following properties:

$\left(1^{\circ}\right)$ it is an E-solution of the equation

$$
\Sigma_{n, r}(1 / x)=b / a, \quad(1<r<(n-1)) ; \dagger
$$

* If the sign $<$ in the second relation below were replaced by the sign $\leqq, X_{1} \ldots(r+1)$ would still be a set $\sigma$ in a sense perfectly analogous to that in which the term set $\sigma$ was used in I.

$\dagger$ The case $r=1$ has been treated in Theorem $\mathrm{A}$; the case $r=(n-1)$, in $\mathrm{I}$. Hence only the cases $1<r<(n-1)$ remain for consideration. 


$$
\Sigma_{p, r}(1 / w)=\frac{b w_{1} \cdots w_{p}-1}{a w_{1} \cdots w_{p}}
$$

for every positive integral value of $p$ for which $\mu \leqq p \leqq(n-1)$ and for no positive integral value of $p$ less than $\mu$;

$\left(3^{\circ}\right)$ if $x=X$, where $X \neq w$, is an E-solution of the equation in $\left(1^{\circ}\right)$, then for every positive integral value of $p$ such that $r \leqq p \leqq \mu$ for which $X_{1 \ldots p} \neq w_{1} \ldots p$, the relation $\Sigma_{p, r}(1 / X) \leqq \Sigma_{p, r}(1 / w)$ holds.

Then $w_{n}$ is the largest number that exists in any E-solution of the equation in $\left(1^{\circ}\right)$ and $w_{n}$ appears in no E-solution of this equation except w.

THEOREM 3a. If $x \neq w$ is any E-solution of the equation considered in Theorem 2a, $P(x)<P(w)$. [See Theorem 3 of I.]

Since the methods which we have used in proving Theorem A together with those of I suffice to prove the last two theorems we omit their proofs.

The following corollary shows that Theorem $i \mathrm{a},(i=2,3)$, has content for a case in which $\mu>r$.

Corollary 4 . The Kellogg solution of the equation $\Sigma_{n, 3}(1 / x)$ $=5 / 11, n \geqq 5$, is $x=w$, where $w_{1}=w_{2}=1, w_{3}=3, w_{4}=14, w_{i+1}$ $=11 \Sigma_{i, i-r+1}(w)+1,(i=4, \cdots, n-2), w_{n}=11 \Sigma_{n-1, n-r}(w)$.

It is easy to show that in this example the $\mu$ of Theorem $2 \mathrm{a}$ exists and equals 4 .

10. Generalizations of Theorems 4,5 .

Theorem 4a. Suppose $a, b, \mu$ are as in Theorem 2a and that there exists $a$ set of $n$ numbers $w>\left(w_{1}, \cdots, w_{n}\right)$ with the following properties:

$\left(1^{\circ}\right) i^{+}$is an E-solution of the equation $\Sigma_{n, r}(1 / x)+\lambda_{r+1}(1 / x)=b / a$, in which $\lambda_{r+1}$ is an integer $\geqq 0$;

$$
\Sigma_{p, r}(1 / w)+\lambda_{r+1} \Sigma_{p, r+1}(1 / w)=\frac{b w_{1} \cdots w_{p}-1}{a w_{1} \cdots w_{p}}
$$

for every positive integral value of $p$ for which $\mu \leqq p<n$ (and for no positive integral value of $p$ less than $\mu$ when $\mu>r$ );

$\left(3^{\circ}\right)$ if $x=X$, where $X \neq w$, is an E-solution of the equation in $\left(1^{\circ}\right)$, then for every positive integral value of $p$ such that $r \leqq p \leqq \mu$ for which $X_{1 \ldots p} \neq w_{1} \ldots p$, the relation 


$$
\Sigma_{n, r}(1 / X)+\lambda_{r+1} \Sigma_{p, r+1}(1 / X) \leqq \Sigma_{p, r}(1 / w)+\lambda_{r+1} \Sigma_{p, r+1}(1 / w)
$$

hoids.

In such a set $w, w_{n}$ is the largest number that exists in any E-solution of the equation in $\left(1^{\circ}\right)$ and $w_{n}$ appears in no E-solution of this equation except $w$. Furthermore a similar statement holds when the left member of the equation in $\left(1^{\circ}\right)$ is replaced by

$$
\Sigma_{n, r}(1 / x)+\Sigma_{n, r+1}(1 / x)+\cdots+\Sigma_{n, s}(1 / x),
$$

where, as heretofore, $s$ is a positive integer and $r<s \leqq n$.

Theorem 5a. In each of the two cases of Theorem $4 \mathrm{a}$, if $X$ is an E-solution of the given equation and $\neq w$, the Kellogg solution of that equation, then $P(X)<P(w)$.

The following corollaries show that the theorems of this section have content in cases where $\mu=2$ when $r=1$.

Corollary 5. For the equation $\Sigma_{n, 1}(1 / x)+3 \Sigma_{n, 2}(1 / x)=5 / 17$, wiih $n>2$, the set $w$ of Theorem $4 \mathrm{a}$ is given by $w_{1}=4, w_{2}=40$, $w_{i+1}=17\left[\Sigma_{i, i}(w)+3 \Sigma_{i, i-1}(w)\right]+1, \quad(i=2, \cdots, n-2), \quad$ and $w_{n}=17\left[\Sigma_{n-1, n-1}(w)+3 \Sigma_{n-1, n-2}(w)\right]$.

Corollary 6. For the equation $\Sigma_{n, 1}(1 / x)+\Sigma_{n, 2}(1 / x)=4 / 13$, with $n>2$, the $w$ of Theorem 4 a (see last sentence of that theorem) is given by $w_{1}=4, w_{2}=22, w_{i+1}=13\left[\Sigma_{i, i}(w)+\Sigma_{i, i-1}(w)\right]+1$, $(i=2, \cdots, n-2)$, and $w_{n}=13\left[\Sigma_{n-1, n-1}(w)+\Sigma_{n-1, n-2}(w)\right]$.

NorthWESTERN UnIVERSITY

\section{ERRATA}

The following changes should be made in the present volume (Vol. 40) of this Bulletin:

Page 93, last line of Theorem 2, insert before the words " $i s$ that" the words "and that $f_{m}(x)$ be continuous."

Pages 413-416, change $f$ to $f_{0}$ in the following places: in the statement of Theorem 2 on p. 413 ; in the statement of Theorem 6 on p. 415 ; and in five places occurring in the first six lines of p. 416. 